\title{
Análisis de la sostenibilidad de los procesos de fortalecimiento de la asociatividad rural: El caso de Asomora
}

\author{
Sustainability analysis of the strengthening processes of a rural association: Asomora a case study
}

\author{
Holmes Rodríguez E. ${ }^{1}$; Carlos Julián Ramírez G. ${ }^{2}$
}

\footnotetext{
1 Profesor Asistente, I. A. Ph. D., Grupo GaMMA, Universidad de Antioquia UdeA. Medellín. Colombia, holmes.rodriguez@ udea.edu.co.

2 Grupo GISER. I. A. M.Sc., Universidad de Antioquia UdeA. Medellín. Colombia.
}

Citar: RODRÍGUEZ, H.; RAMÍREZ, C. 2016. Análisis de la sostenibilidad de los procesos de fortalecimiento de la asociatividad rural: El caso de Asomora. Rev. Cienc. Agr. 33(1): 9 - 21.

Fecha recepción: Agosto 03 de $2015 . \quad$ Fecha de aceptación: Noviembre 11 de 2015.

\section{RESUMEN}

En Colombia, se han implementado diversas políticas públicas de apoyo a la organización de productores rurales, como estrategia para mejorar el acceso al mercado de los pequeños productores, con resultados poco satisfactorios para el esfuerzo realizado. El objetivo de éste estudio fue identificar los factores relacionados con el logro de la sostenibilidad de los procesos de fortalecimiento de la asociatividad rural. Se realizó una investigación cualitativa a partir del estudio de caso de la Asociación de Productores de Mora (Asomora) de la localidad de Trujillo en el departamento del Valle del Cauca. El proceso de fortalecimiento de la asociación, tuvo como logro más importante el incremento en el ingreso del productor por el acceso directo al mercado. Sin embargo, el proceso no logró alcanzar la sostenibilidad de la organización debido principalmente al individualismo, bajo compromiso de los productores con la organización y falta de capital financiero para competir con los intermediarios. Los resultados de este estudio permiten concluir que para el logro de la sostenibilidad de los procesos de asociatividad rural, se deben abordar de manera integral aspectos: económicos, técnicos, ambientales, políticos y sociales, que permitan garantizar la construcción de capital social, por medio de relaciones de confianza entre los asociados y su compromiso para el logro de los planes construidos de manera participativa, mediante la acción colectiva.

Palabras clave: organización, productores, acceso, mercado, capital social. 


\begin{abstract}
In Colombia, different public policies have been implemented to support small rural producers as a strategy to improve their access to markets showing low satisfactory results. The aim of this paper was to identify the factors related to the achievement of the sustainability of the strengthening processes of the rural associativity. A qualitative research was carried out with the case study of the Association of Small Producers of Blackberry (Asomora), in the municipality of Trujillo, state of Valle del Cauca. The strengthening process of Asomora showed as the most significant achievement the increase in the direct market-access rate. However, the process did not reach the sustainability of the Association due to self-interest issues, low commitment of the produces to the Association and lack of financial funds to compete with intermediaries. The results of this study showed that to reach sustainability in rural associativity processes, economic, technical, environmental, social and political factors must be integrally and simultaneously analyzed to guarantee the proper construction of social capital through confidence relations between the associations' members and their commitment to build participative plans..
\end{abstract}

Key words: organization, producers, access, market, social capital.

\section{INTRODUCCIÓN}

En Colombia, las políticas públicas para el financiamiento de las actividades agropecuarias, tienen como uno de sus ejes, los fondos parafiscales, las contribuciones obligatorias por parte de los productores que tienen una destinación especial (Plata et al., 2010) entre la cual se encuentra el mejoramiento del acceso de los pequeños productores al mercado. La legislación que regula estos fondos parafiscales, estableció que deben ser administrados por organizaciones gremiales, actividad por la cual reciben una contraprestación que contribuye a su sostenibilidad económica, situación que favoreció el florecimiento de gremios nacionales por subsectores, entre los cuales se encuentran: porcicultores, ganaderos, hortifruticultores, cerealistas, arroceros, palmicultores, papicultores, paneleros, cacaoteros, caficultores.

No obstante, la existencia de éstos instrumentos de fomento a la producción agropecuaria que favorecen la gremialidad, en el sector, persisten problemas de baja asociatividad que traen como consecuencia, deficiencias en la optimización del uso de los recursos productivos para mejorar las condiciones socioeconómicas de los productores (Sociedad de Agricultores de Colombia, 2010) con las consecuentes limitaciones para el desarrollo agropecuario nacional. Para el caso de las frutas y hortalizas, el gremio nacional fue creado en 1993 y tiene a su cargo la administración del Fondo Nacional de Fomento Hortifrutícola, creado por la Ley 118 de 1994 (Congreso de Colombia, 1994).

Sin embargo, la complejidad del subsector hortifrutícola constituido principalmente por pequeños productores, de más de 70 especies de frutas y más de 80 de hortalizas, con una producción dispersa por todo el país, ha ocasionado que persistan problemas como la baja asociatividad y el bajo nivel de empresarización (ASOHOFRUCOL, 2012). Adicionalmente, los canales de distribución del subsector hortifrutícola están integrados por un alto número de intermediarios que no agregan valor al productor, encareciendo el costo de distribución, lo cual afecta tanto al precio que paga el consumidor como la rentabilidad del productor primario; se tiene además, un sistema de distribución mayorista integrado por pocos compradores, en el cual los productores por su bajo nivel de asociatividad, tienen muy poco poder de negociación y los precios de compraventa son definidos por el mayorista. Al respecto, las cifras en países en desarrollo, muestran como las cooperativas de productores contribuyen con más del $50 \%$ de las cosechas de 
productos agrícolas y para el caso de frutas y hortalizas en países como Holanda y Bélgica contribuyen con más del 80\% de las cosechas (Bijman, 2013).

La asociatividad es un proceso de trabajo colectivo o cohesión social, en busca de objetivos comunes (Bolaños, 1999) definidos por el grupo, a partir del desarrollo de principios y valores como confianza, compromiso, participación, liderazgo y comunicación (Amézaga et al., 2013) para la obtención de resultados, que de forma individual son más difíciles de alcanzar en aspectos productivos, organizativos y comerciales, haciendo un uso más eficiente de los recursos naturales, humanos, sociales, físicos y financieros disponibles (Gottret et al., 2011). Por lo anterior, es fundamental para el fortalecimiento de esquemas asociativos, el mejoramiento de las relaciones interpersonales (Centro para el Desarrollo de Capital Humano, 2007), al igual que el análisis de estructuras sociales, capital social y cohesión de la población beneficiaria (Efendiev y Sorokin, 2013).

Entre las ventajas de la asociatividad reportadas en la literatura científica se tienen: mayor facilidad de acceso a asesoría técnica para la producción, acceso a materias primas más económicas, mayor facilidad en el acceso a servicios financieros (Álvarez et al., 2012), incremento de los márgenes económicos, mayor capacidad de enfrentar las amenazas del entorno, contribución al desarrollo socioeconómico por medio de la construcción de capital social y capital humano (Amézaga et al., 2013).

Por otra parte, algunas de las limitaciones al desarrollo asociativo reportadas en la literatura, son la falta de planes estratégicos de desarrollo de la organización, deficiencias en la gestión administrativa y contable, debilidades en la capacitación de la base social (Ostertag, 2002), la baja participación de los productores en los procesos organizativos (Riella y Vitelli, 2009), la dificultad para el trabajo en equipo, deficiencia en la distribución de funciones y en la toma colectiva de decisiones (Szmulewicz et al., 2012).
Teniendo en cuenta la importancia de la organización de los productores para el desarrollo sectorial, el gobierno nacional ha implementado desde finales del siglo XX, diversos programas de apoyo al fortalecimiento de procesos asociativos; en el Valle del Cauca uno de ellos fue el Programa Regional de Mercadeo Agropecuario (Rodriguez et al., 2008) en el cual participó la Asociación de Productores de Mora (Asomora) localizada en el municipio de Trujillo, que a pesar del acompañamiento recibido por el mencionado programa, al igual que apoyos posteriores a lo largo de una década, no logró consolidar su proceso organizativo, sin ser claras las causas por las cuales no se tuvieron los resultados esperados.

Ante esta necesidad de comprender los factores que inciden en el éxito de los procesos organizativos, se plantea el concepto de sostenibilidad asociativa como una propuesta para analizar el grado de fortalecimiento que alcanza una organización para subsistir de manera autónoma, una vez finaliza el proceso de acompañamiento institucional. Tal concepto, se construye a partir de la teoría de las tres dimensiones del desarrollo sostenible: económica, social y ecológica (Artaraz, 2001); al igual que la dimensión político-institucional planteada por el IICA en la multidimensionalidad del desarrollo sostenible (Sepúlveda et al., 2002) y por último, en la dimensión técnica como base del proceso productivo de los integrantes de la organización.

En éste sentido, el concepto de sostenibilidad asociativa que se plantea, considera en su componente social, variables como las habilidades y destrezas del recurso humano y su grado de compromiso. En su componente económico, la capacidad de generación de ingresos adecuados para el productor y para el sostenimiento de la organización y la prestación de servicios a sus asociados. En el componente institucional, la participación de los productores en la toma de decisiones en la organización y de la organización en los instrumentos de política pública. En el componente técnico, la prestación de servicios de asistencia técnica para mejorar la eficiencia de los sistemas de 
producción y en el componente ambiental, las estrategias de mitigación del impacto de los sistemas productivos sobre el agua, el suelo y los bosques.

Por ésta razón, el objetivo de éste estudio fue identificar los factores relacionados con el logro de la sostenibilidad de los procesos de fortalecimiento de la asociatividad rural a partir del estudio del caso de Asomora, con el fin de identificar aspectos que permitan orientar las políticas públicas de fortalecimiento de la asociatividad.

\section{MATERIALES Y MÉTODOS}

Se realizó una investigación de carácter exploratorio (Lamnek, 2005; Noelle y Petersen, 2005), utilizando la técnica de estudio de caso (Martínez, 2006), con las siguientes etapas: selección del caso de estudio para estudiar el fenómeno de interés, los factores que inciden en la sostenibilidad de la asociatividad rural; identificación de informantes clave de la organización y relacionados con ella, diseño de los instrumentos de recolección de la información necesaria para el estudio del caso en sus aspectos histórico, social, administrativo, técnico, comercial, político y económico, recolección y análisis de la información, comparación de los resultados del caso estudiado con la literatura científica y finalmente, la formulación de conclusiones e implicaciones del estudio.

Para la selección de la organización objeto de estudio, se definieron los siguientes criterios: integrada por pequeños productores, participación en varios programas de apoyo al fortalecimiento asociativo, permanencia en el tiempo y disponibilidad de información sobre la organización.

A partir del cumplimiento de estos criterios, se seleccionó la Asociación de Productores de Mora del municipio de Trujillo, Valle del Cauca; ubicado en el centro del Valle del Cauca a $116 \mathrm{~km}$ de la ciudad de Cali, principal centro de consumo de la región. El municipio tiene una superficie total de $222 \mathrm{~km}^{2}$ distribuidos desde los 1000 hasta los $2000 \mathrm{msnm}$, con una altitud promedio de $1260 \mathrm{msnm}$ y una temperatura promedio de $21^{\circ} \mathrm{C}$. La zona de producción de la Asociación es la vereda Chuscales, ubicada en la cordillera occidental entre los $1800 \mathrm{y}$ $2000 \mathrm{msnm}$. Las fincas se encuentran en una zona hasta donde no llegan carreteras, con recorridos en mula de 2 a 4 horas de duración hasta la vía interveredal donde llegan los vehículos para realizar el acopio de la producción.

El análisis preliminar de la organización, por medio de visitas de reconocimiento y observación, permitió identificar como informantes clave: 10 productores, tres integrantes del personal administrativo de la asociación, tres funcionarios de las instituciones de apoyo (Unidad Municipal de Asistencia Técnica Agropecuaria, Secretaría de Agricultura del Valle del Cauca, Central de Cooperativas Agrarias), tres intermediarios y tres almacenes de cadena de la ciudad de Cali.

Con los informantes clave identificados, se diseñaron entrevistas semiestructuradas como instrumentos de recolección de información. Estas se llevaron a cabo en el período enero 2012 a diciembre de 2014. La información primaria recolectada se transcribió para realizar el proceso de consolidación y comparación constante con la literatura científica analizada, acorde con el objeto de estudio.

\section{RESULTADOS Y DISCUSIÓN}

El proceso de fortalecimiento de la asociatividad rural de Asomora permitió identificar tres momentos, en la línea de tiempo, el origen del proceso organizativo informal, la consolidación del proceso organizativo formal y la autogestión para el logro de la sostenibilidad (Tabla 1). Se encontró además, que el proceso se centró en la dimensión económica por medio del mejoramiento de las condiciones de los productores de acceso al mercado. 
Tabla 1. Fases del proceso organizativo de Asomora.

\begin{tabular}{llll}
\hline \multicolumn{1}{c}{ Etapa } & Período & $\begin{array}{r}\text { Tipo de } \\
\text { proceso }\end{array}$ & \multicolumn{1}{c}{ Dimensión atendida } \\
\hline Origen de la asociación & $1986-1991$ & Informal & Económica-Social \\
Consolidación del proceso organizativo & $1992-1997$ & Formal & Económica \\
Autogestión & $1998-2008$ & Formal & Económica-Técnica \\
\hline
\end{tabular}

Origen de la asociación. La producción de mora en la vereda Chuscales, inició en la década del 70, en una zona de reserva forestal, como etapa posterior a la extracción de madera y agricultura de subsistencia, como resultado de un ensayo exitoso por parte de un agricultor, lo cual ocasionó, que todos los productores de la vereda cambiaran la actividad maderera por la producción de mora con pequeñas áreas cultivadas debido principalmente a problemas en la tenencia y acceso a capital de trabajo para la compra de insumos. En esta etapa, los productores realizaban la venta de su producto de manera individual en la vereda, a un intermediario en guacales de madera con peso promedio de $15 \mathrm{~kg}$ de mora por los cuales solo recibían un pago estándar sin tener en cuenta el peso real, equivalente a $12,5 \mathrm{~kg}$. El desconocimiento del mercado y la falta de acceso a la información de precios, ocasionaba que los productores no tuvieran ningún poder de negociación y que el precio fuera establecido de manera unilateral por el intermediario.

Como resultado de una visita realizada por un grupo de productores al mercado final en la ciudad de Cali, en la cual encontraron que el precio de compra del mayorista era tres veces mayor al precio de venta del productor, se produce un cambio en el sistema de distribución. Tres productores deciden alquilar un vehículo y sacar su producción directamente al mercado terminal. Teniendo en cuenta que los productores de mora de la zona no utilizaban productos químicos como insumos de la producción, por falta de recursos, la calidad de la mora fue un factor reconocido por los mayoristas que incidió en una rápida penetración del mercado y el inicio del proceso organizativo.
Como resultado de esta incursión al mercado terminal y por consiguiente, el acortamiento del canal de distribución, el margen de utilidad bruta de estos productores aumentó y rápidamente los otros productores de la vereda se unieron a este naciente canal de comercialización, dando origen en 1986 a la conformación de un grupo informal constituido por 50 productores, que un año más tarde dió origen al proceso organizativo, por medio de la constitución de la Asociación de Productores de Mora con 187 asociados (Asomora).

En 1991, Asomora comercializaba en promedio, $75 t$ anuales de mora, que representaban 35\% de la producción municipal, de las cuales $80 \%$ tenían como destino el mercado fresco y $20 \%$ la industria procesadora. El fortalecimiento del trabajo grupal, trajo consigo la competencia del intermediario por no salir de la zona; en épocas de escasez realizaba prácticas como mayor pago al productor por kilo de producto, pagos en efectivo al momento de la compra, pagos por adelantado antes de la cosecha, entre otros.

En esta fase del proceso, los productores se enfrentaron a otro reto, el alto costo del transporte. A pesar de la cercanía de la zona de producción en Trujillo con el mercado terminal de la ciudad de Cali, $150 \mathrm{~km}$, la dificultad en el acceso a la vereda por el mal estado de la vía y la poca oferta de servicio de transporte, encarecían el costo. Teniendo en cuenta esta problemática y como estrategia para mejorar las condiciones de vida en la zona, afectada para la época, por fenómenos de violencia, la iglesia católica del municipio ayudó a la asociación a gestionar la consecución de un 
camión. Sin embargo, el medio de transporte no solucionó el problema por cuanto se generaron dificultades al interior de la organización por el manejo del vehículo, problemas con el conductor $\mathrm{y}$ en especial la inexistencia de un fondo para reparación y reposición del vehículo, por lo cual finalmente el vehículo tuvo que ser vendido.

Consolidación de la organización. En 1992, la Central de Cooperativas Agrarias (CENCOA), organización cooperativa de segundo nivel del gremio cafetero, inicia la implementación del plan regional de mercadeo agropecuario (PRMA) con recursos del fondo para el Desarrollo Rural Integrado (DRI), siendo Asomora una de las organizaciones de productores beneficiarias. Uno de los efectos que tuvo el acompañamiento de CENCOA al proceso de comercialización, fue la implementación de la compra por peso para garantizar equidad en la comercialización reemplazando el sistema de compra por guacal, con un peso estimado de $12,5 \mathrm{~kg}$, a un sistema más justo mediante la compra por kilo, con base en una medida objetiva. La implementación de este cambio, obligó al intermediario a cambiar también su sistema de compra por medio del pesaje, lo cual contribuyó al mejoramiento de los ingresos de los productores de la zona, incluyendo los no pertenecientes a Asomora. El ingreso de los productores como resultado de la gestión comercial de la asocia- ción se incrementó, logrando alcanzar en promedio 2,5 salarios mínimos mensuales vigentes.

Otro de los aspectos que mejoró durante esta fase fue el mercado terminal; Asomora pasó de vender toda su producción en el mercado mayorista, a vender a la industria procesadora por medio de acuerdos informales de abastecimiento y a los supermercados, lo cual representó un incremento en el precio de venta. Esto permitió también la generación de empleo, principalmente de mujeres, para las labores de selección y clasificación del producto con destino al mercado especializado. Por otro lado, en esta fase se inició también la venta directa a los consumidores del municipio, en un punto de venta localizado en el centro de acopio.

Esta nueva etapa del desarrollo de la organización, obligó a la contratación de personal para atender el mercado final y para el manejo de los recursos y las cuentas de la organización, lo cual implicó el establecimiento de deducciones en el precio de venta para cubrir los costos de personal, lo cual representaba una necesidad de aumentar los volúmenes de acopio y comercialización para cubrir los costos de funcionamiento, enfrentándose la organización a varias limitaciones (Tabla 2).

Tabla 2. Principales dificultades presentadas en el proceso organizativo.

\begin{tabular}{ll}
\hline \multicolumn{1}{c}{ Dimensión } & \multicolumn{1}{c}{ Dificultad } \\
\hline Económica & Bajos volúmenes de comercialización para cubrir los costos operativos \\
Económica & $\begin{array}{l}\text { Bajos márgenes de utilidad } \\
\text { Ealta de recursos financieros para competir con el intermediario en las compras en } \\
\text { efectivo o pagos por adelantado }\end{array}$ \\
Económica & $\begin{array}{l}\text { Baja capacidad operativa para atender los requerimientos en cuanto tiempos de } \\
\text { entrega de los supermercados }\end{array}$ \\
Social & Falta de construcción de capital social para aumentar el compromiso \\
\hline
\end{tabular}


En 1995, la utilidad neta requerida por Asomora en el proceso comercial para lograr el punto de equilibrio era del 10\%, sin embargo, la utilidad obtenida no superaba el 1\%, como consecuencia de los volúmenes comercializados no fueron suficientes para cubrir estos costos. Los intermediarios con el fin de mantener su capacidad de compra, emprendieron campañas para enfrentar a la asociación ofreciendo en épocas de escasez, adelantos en dinero a los productores, pagaderos al momento de la cosecha y hasta 1000 pesos más en el precio de compra de la caja de mora, con lo cual muchos productores dejaron de entregar el producto a la Asociación, ocasionando mayores problemas en el acopio de los volúmenes necesarios para lograr el punto de equilibrio. Sin embargo, en épocas de abundancia, en las cuales el precio se reduce y se dificulta la venta del producto por la saturación del mercado terminal, los intermediarios pagaban al productor precios muy bajos y en ocasiones no compraban las cosechas, por lo cual los productores entregaban la producción a la Asociación, ocasionando a ésta, dificultades para la venta de volúmenes de producto superiores a los tradicionales.

Otra dificultad que surgió en esta etapa, fue que los agricultores estaban acostumbrados al pago en efectivo de su producto, por cuanto las ventas se hacían a intermediarios y mayoristas que pagaban en efectivo, sin embargo, la venta a los supermercados, implicaba pagos a las semanas. Esta situación ocasionó que muchos agricultores prefirieran la venta en efectivo al intermediario, aunque el precio fuera inferior al de la Asociación, por lo cual muchos productores empezaron a dejar de participar en la comercialización a través de Asomora.

En este mismo proceso de competencia, los intermediarios, al contar con cuartos de enfriamiento en la ciudad de Tuluá, sitio en el cual podían acopiar mora de otras veredas diferentes a Chuscales y de otros municipios productores, lo cual les permitía tener mayores volúmenes y más continuidad en el abastecimiento, se volvieron también proveedores de los supermercados, logrando mayor capacidad comercial que la Asociación. Esta mayor capacidad operativa de los intermediarios, les permitió además abastecer el mercado mayorista y el de las industrias con el producto que no cumplía los estándares de calidad de los supermercados, convirtiéndose en un fuerte competidor de la asociación en el mercado terminal.

Por otro lado, las estrechas relaciones que fueron construyendo el gerente y el conductor de la asociación con los compradores y la mayor rentabilidad de la comercialización de la mora frente a su producción primaria, ocasionó que ellos abandonaran la Asociación para convertirse en intermediarios y competidores de ella. Otro factor que afectó a Asomora en esta fase, fue la política de devolución del producto implementada por los supermercados, los cuales realizaban descuentos al pago, a la Asociación, por cuenta del producto deteriorado en el supermercado luego de permanecer en exhibición.

Tabla 3. Principales dificultades presentadas en el proceso organizativo.

\begin{tabular}{|c|c|}
\hline Dimensión & Dificultad \\
\hline Técnica & $\begin{array}{l}\text { Deficiencias en la prestación de servicio de asistencia técnica en producción primaria } \\
\text { de mora }\end{array}$ \\
\hline Social & $\begin{array}{l}\text { Reducción en el número de socios activos por falta de confianza en los directivos de la } \\
\text { organización }\end{array}$ \\
\hline Ambiental & Degradación del recurso suelo por ausencia de prácticas de conservación \\
\hline Político & Reducida participación de los productores en la toma de decisiones en la organización \\
\hline Económico & Deficiencia en la gestión administrativa para el cumplimiento de las metas \\
\hline
\end{tabular}


Sostenibilidad de la organización. En 1998, terminó el apoyo que recibía la organización por parte de CENCOA, lo trajo consigo el aumento de las dificultades para la organización (Tabla 3).

La falta de apoyo sumada a la reducción en la producción, ocasionó un descenso en las ventas afectando la confianza de los productores en la organización con los consecuentes problemas en las finanzas de Asomora, lo cual condujo a una crisis económica que afectó la estabilidad de la organización. Adicionalmente, se presentaron problemas entre los integrantes de la asociación y se redujo la confianza en los directivos de la organización.

La crisis de la asociación llevó a la reducción del número de productores que comercializaban su producción a través de ella, llegando a solo cuatro. No obstante, debido a las ventajas de la zona de producción de Chuscales, en cuanto a los grados brix del producto y el cultivo sin utilización de productos químicos, se logró el apoyo de una organización no gubernamental (ONG) para fortalecer la asociación. Este nuevo acompañamiento a la asociación, permitió lograr un acuerdo informal de proveeduría con una empresa privada con fines de procesamiento industrial, de esta manera Asomora pasó de acopiar 3,5 a 6t/semana, recuperando la confianza de sus asociados, aumentando de nuevo el número de socios activos hasta llegar a 14 .

En 1999, el apoyo de la ONG en la gestión administrativa de Asomora, permitió evidenciar problemas financieros de la asociación y problemas productivos de sus asociados, por lo cual se inició un proceso de asistencia técnica para el mejoramiento de la producción primaria; prácticas como la fertilización, realización de podas y renovación de cultivos no eran realizadas por los productores por lo cual la productividad era muy baja. A pesar que las recomendaciones técnicas indican la necesidad de renovar el cultivo cada seis años, los agricultores habían cultivado sin renovar durante 15 años, situación por la cual la productividad de la vereda Chuscales era inferior a 4t/ha, muy baja si se compara con la productividad esperada (14t/ha) y la productividad media nacional (10t/ha). La renovación de los cultivos permitió entonces aumentos de la productividad, llegando a 8t/ha.

En el 2002, otra ONG con recursos del Ministerio de Agricultura y Desarrollo Rural, emprendió la tarea de creación de una organización de segundo grado para agrupar a las asociaciones de productores de mora de los cinco municipios de mayor producción del departamento del Valle del Cauca. Asomora quedó incluida en este proceso que finalizó con la constitución de la Asociación de Familias Cultivadoras de Mora (ASOFAMORA) integrada por 120 productores.

ASOFAMORA logró acuerdos comerciales para la venta de 1800 toneladas mensuales de mora, $70 \%$ con destino a empresas procesadoras y $30 \%$ con destino a supermercados regionales. ASOFAMORA acopiaba la producción en la ciudad de Tuluá, equidistante a las zonas de producción y posteriormente la enviaba al centro de consumo más importante de la región, la ciudad de Cali. Por realizar este proceso, descontaba a las organizaciones de productores el $5 \%$ del valor de las ventas, como costos de comercialización; este sistema de comercialización era muy atractivo para las nacientes organizaciones de productores de otros municipios, pero no para Asomora que tenía una experiencia superior a 15 años en la comercialización de la mora, por lo cual esta asociación, dejó de comercializar la producción de sus asociados a través de ASOFAMORA y retornó a sus anteriores canales de comercialización, convirtiéndose en competidor de ASOFAMORA.

La política nacional de apoyo a la organización regional de productores de mora, ocasionó incrementos en las áreas sembradas y por consiguiente en la producción regional, por lo cual para Asomora fue más difícil competir y sus problemas de acceso al mercado persistieron. 
En el 2003, la producción de mora del departamento del Valle del Cauca alcanzó las 5588 toneladas, de las cuales el municipio de Trujillo contribuyó con 2002Tn (36\%), no obstante, el acopio realizado por Asomora solo alcanzó las 48 toneladas.

En el 2007, Asomora continuó con sus 14 socios, los cuales por problemas de tecnificación de la producción, vieron su producción reducida de un promedio de $1 \mathrm{t} / \mathrm{mes}$ a $100 \mathrm{~kg} / \mathrm{mes}$, situación que llevó a los productores a tener que emplear su fuerza de trabajo en otras explotaciones para lograr un ingreso suficiente para cubrir sus costos de vida. Por esta razón, Asomora solo alcanzó un acopio de 1,8t/mes, con lo cual su capacidad de negociación se vio muy disminuida, regresando al sistema de comercialización de 20 años atrás a través de intermediarios que venden en el mercado mayorista, muy inestable y con baja rentabilidad para los productores.

Los resultados de éste estudio, permiten evidenciar la potencialidad que tiene la acción colectiva para mejorar las condiciones de los pequeños productores en el acceso al mercado, lo cual coincide con los hallazgos de otros estudios, que plantean como uno de los retos de la promoción de la asociatividad el logro de la adopción de un enfoque de mercado por parte de las organizaciones que permita a sus asociados beneficiarse de las economías de escala generadas de la acción colectiva (Amézaga et al., 2013). Sin embargo, el fortalecimiento organizativo de Asomora se concentró en la dimensión económica dejando de lado las otras dimensiones de la sostenibilidad asociativa, lo cual pone de manifiesto las dificultades y la complejidad que implica este tipo de procesos.

A pesar que la literatura reporta que los procesos organizativos que surgen de las necesidades de los beneficiarios tienen mayores posibilidades de éxito que aquellos orientados solo a cumplir metas cuantitativas de instituciones oficiales (Medici, 2006), en el caso de Asomora el proceso organizativo surgió de la motivación de los productores para atender la necesidad de mejorar el acceso al mercado y permitió logros parciales en este aspecto, sin embargo, el logro global en el fortalecimiento de la organización, fue poco satisfactorio.

En términos generales, la participación de los productores en las diferentes etapas del proceso organizativo fue reducida al compararse con el número elevado de productores que iniciaron el proceso. Estos hallazgos concuerdan con otros estudios que reportan la baja participación de los productores tanto en los espacios administrativos como en la toma de decisiones (Álvarez et al., 2012), relacionada con baja confianza en las relaciones entre los asociados, con una percepción de los asociados de sus organizaciones como un intermediario comercial en el cual ellos se sienten más como un proveedor que como integrantes de la organización.

En el mismo sentido, otros autores (Szmulewicz et al., 2012) han encontrado que los productores, pasan de una fase de alto compromiso a otra de vínculo comercial; en esta última fase, el nivel de cohesión social es muy bajo y la motivación principal está relacionada con los beneficios económicos que se pueden lograr a nivel individual. A pesar que otros autores (Lattuada et al., 2011) han encontrado que el crecimiento de las organizaciones ocasiona una pérdida de la identificación de los asociados con el proyecto organizativo, para el caso de Asomora la misma pérdida de identificación se dio antes que la organización tuviera un crecimiento notable.

La falta de atención al fortalecimiento socio-organizacional, fue una debilidad grande del proceso, por cuanto como lo expresan otros autores (Amézaga et al., 2013) éste es un elemento fundamental para la generación de relaciones de confianza, compromiso, comunicación y colaboración entre los productores con una definición clara de los objetivos por los cuales se organizan y una visión de futuro compartida; estos aspectos son complementarios al fortalecimiento económicoproductivo. En este mismo sentido, otros estudios (Szmulewicz et al., 2012) han encontrado que el desarrollo exitoso de una organización asociativa, 
depende de la participación activa de sus integrantes por medio de reuniones periódicas y la distribución de funciones.

Por otro lado, Bolaños (1999) reporta la importancia de caracterizar los grupos de beneficiarios para lograr procesos de fortalecimiento socio-organizacional, teniendo en cuenta aspectos como las razones que tienen para organizarse, las características de sus sistemas de producción, los mecanismos de comunicación que utilizan entre ellos, su grado de cohesión como comunidad y las formas de organización que tienen. De la misma forma, el análisis de los beneficios de la acción colectiva, inspección de la motivación para el trabajo en grupo (Centro para el Desarrollo de Capital Humano, 2007) y la elaboración de instrumentos orientados al desarrollo sostenible como el plan estratégico, el plan de negocios y el plan operativo (Amézaga et al., 2013).

Un aspecto que merece una especial atención, es que a pesar de todo el apoyo recibido por Asomora de los instrumentos de política pública como el Fondo DRI y de la Alcaldía Municipal no logró su sostenibilidad. Al respecto, los hallazgos de otros autores Efendiev y Sorokin (2013) resaltan la importancia de las ayudas estatales para proveer considerable ayuda tecnológica, administrativa, gerencial y financiera a los organizaciones de productores para su desarrollo sostenible.Una de las explicaciones a estos resultados paradójicos, puede ser la planteada por otros estudios (Medici, 2006) en cuanto que la acción colectiva es un proceso complejo que requiere una intervención prolongada para lograr desarrollar la confianza entre los asociados necesaria para llegar a la acción grupal por el beneficio común, así como también la responsabilidad, habilidades comunicativas, trabajo en equipo y toma de decisiones de manera colectiva (Szmulewicz et al., 2012), aspectos que no se trabajaron en Asomora.

Otro factor que puede explicar la deficiencia en el fortalecimiento organizativo de Asomora, es el bajo nivel educativo de los líderes, en su mayoría con nivel de escolaridad primaria y analfabetas, por cuanto de acuerdo con los hallazgos de otros estudios (Ostertag, 2002) el éxito de procesos organizativos, ha estado relacionado con la presencia de un líder con buen nivel educativo y habilidades empresariales. El bajo nivel educativo de quienes lideraron la asociación, pudo haber incidido en debilidades en las habilidades empresariales necesarias para la implementación de estrategias conducentes al aumento de los volúmenes de acopio y comercialización y el cubrimiento de los costos de funcionamiento.

El bajo nivel educativo puede explicar también la baja participación de los productores en el proceso organizativo de Asomora, por cuanto de acuerdo con los hallazgos de otros autores (Szmulewicz et al., 2012) es un factor que incide en su baja participación en procesos de capacitación y de toma de decisiones. Por otra parte, Américo et al. (2013) indican que involucrar a los productores por medio de metodologías participativas en la identificación de necesidades y el planteamiento de alternativas de solución, contribuye no solo a elaborar planes ajustados a las necesidades de los beneficiarios, sino también a mejorar su compromiso con los procesos de intervención.

Igualmente, como lo plantea Medici (2006), la ayuda mutua se intensifica cuando existen reglas adecuadas al interior de la organización para evitar acciones oportunistas por parte de los asociados que van en detrimento de la organización. En este sentido, los hallazgos de otros autores (Álvarez et al., 2012), plantean algunas estrategias que contribuyen al logro de un compromiso del asociado con la sostenibilidad económica de su organización, es la comercialización del 100\% de su producción a través de la asociación, con la finalidad de fortalecer su capacidad de negociación y evitar conflictos a la hora de decidir si entrega la producción a la asociación o al intermediario. Igualmente, el mejoramiento de la calidad de manera que el asociado optimice el uso de los servicios como insumos y asesoría técnica para obtener productos de la mejor calidad y que 
este esfuerzo sea retribuido mediante el pago del producto acorde con su calidad.

Otro de los aspectos que incidió en el poco éxito del fortalecimiento de la Asociatividad fue la deficiencia en la prestación de servicios a partir de la identificación de demandas por parte de los productores y la evaluación de los servicios ofrecidos. Si bien es cierto que la asociación brindó a sus asociados apoyo a la comercialización, los resultados de (Amézaga et al., 2013) indican que apoyos a la producción y al desarrollo social, son importantes para mejorar el compromiso de los productores, por medio de la evidencia de la mejora en el bienestar de ellos y sus familias así como la promoción del desarrollo de capacidades como el liderazgo y la acción colectiva. Del mismo modo, la falta de capital financiero de la Asociación para pagar en efectivo, fue otro aspecto que atentó contra el compromiso de los productores por cuanto al tratarse de agricultura de subsistencia, sus necesidades básicas no podían esperar los plazos de pago de la Asociación.

A pesar de los beneficios recibidos por los productores como resultado de la existencia de Asomora, como la implementación de un sistema de pago más justo pasando de la compra por caja a la compra por kilo y el mejoramiento de los ingresos de los productores por la optimización del canal de comercialización, la dependencia generada con el intermediario, impidió que estos factores tuvieran un impacto en el compromiso de los productores hacia su asociación. Estos resultados concuerdan con los hallazgos Rincón et al. (2004) quienes encontraron que la alta dependencia del intermediario, es uno de los factores que afecta la participación de los productores en procesos de organización.

Por otra parte, el proceso de fortalecimiento asociativo tuvo deficiencias en la realización de procesos de autoevaluación que permitieran a los asociados analizar el cumplimiento de los objetivos establecidos en la conformación de la organización y de los planes de acción elaborados para cada período. Al respecto, Gottret et al. (2011) plantean como componentes de un sistema de autoevaluación, aspectos como la orientación estratégica; la estructura organizativa, la gestión empresarial, los procesos organizativos, la oferta de servicios de la Organización y la equidad de género.

La organización de los productores, debe ser un componente importante de la política pública por cuanto de acuerdo con los hallazgos de Melgarejo et al. (2013) el desarrollo sostenible de la micro, pequeña y mediana empresa (Mipyme) contribuye al desarrollo regional, especialmente por la generación de empleo y por su aporte al PIB. Adicionalmente, Rueda et al. (2011) han encontrado que los procesos sociales permiten el intercambio y adopción de conocimientos especialmente entre los actores sociales con mayor confianza, reciprocidad y cooperación. Por lo tanto, los resultados poco satisfactorios de éste proceso de fortalecimiento asociativo, pueden servir para orientar la definición de políticas públicas encaminadas a la implementación de procesos sostenibles de asociatividad.

Al respecto, otros estudios (IICA, 2009; Rincón et al., 2004) han encontrado como factores que favorecen la creación de lazos de familiaridad y confianza, los procesos de capacitación de media y larga duración, así como también la generación de resultados parciales en el corto plazo como estrategia para mantener el interés de los productores en la organización; adicionalmente, la implementación de programas de comunicación para mantener informados a los productores sobre el comportamiento de los precios y los mercados (Rincón et al., 2004). En el mismo sentido, la comunicación efectiva que implica el diálogo, el debate de ideas y la toma consensuada de decisiones, permite que los asociados participen de manera activa en el proceso asociativo por medio de la generación de un ambiente de identificación de los productores con su organización lo cual contribuye a la cohesión social (Moreno et al., 2011). 
Rodríguez y Ramírez - Asociatividad rural.

Uno de los aspectos que no se indagó en este estudio, fue la actitud de los productores hacia la participación en el proceso organizativo, por lo cual se plantea la necesidad de realizar nuevos trabajos que profundicen en el entendimiento de las relaciones entre los productores. En este sentido, otros autores han reportado como factores que inciden en el trabajo colectivo, la confianza y compromiso (Amézaga et al., 2013); la cohesión social (Efendiev y Sorokin, 2013) y la deficiencia en la distribución de funciones (Szmulewicz et al., 2012).

\section{CONCLUSIONES}

A partir del análisis de Asomora se puede concluir que un proceso de fortalecimiento de organizaciones de productores rurales debe contemplar la intervención prolongada en el territorio para la implementación de procesos integrales que no solo atiendan el componente económico sino también el social, para mejorar la sostenibilidad social del proceso mediante relaciones de confianza entre los productores y su compromiso y participación activa, bases fundamentales para mejorar la cohesión social y desarrollar habilidades de colaboración para aprovechar las ventajas de la acción colectiva. Adicionalmente la sostenibilidad económica de la organización debe estar soportada en mecanismos claros de generación de ingresos para la prestación de servicios que permitan mantener el interés de los productores en la organización.

\section{REFERENCIAS BIBLIOGRÁFICAS}

ÁLVAREZ, L.; SAIZ, J. E.; DÍAZ, R. D.; CASTILLO, D.; HERRERA, A. 2012. La cooperativa de productores agropecuarios de Lenguazaque, el Valle de Ubaté y municipios circunvecinos (COOPALAC) y su impacto en el desarrollo rural del municipio de Lenguazaque, Cundinamarca. Gest. Soc. 5(2):51 - 73.

AMÉRICO, M.; AOKI, P.; BOSISIO, F.; AMORIM, B.; FONTES, L. 2013. A extensão rural na prática organizativa dos agricultores familiares da comunidade do Córrego do Mosquito, Jaguaré - ES. Cadernos de Agroecología. $8(2): 1$ - 4 .

AMÉZAGA, C.; RODRÍGUEZ, D.; NÚÑEZ, M.; HERRERA, D. 2013. Orientaciones Estratégicas para el Fortalecimiento de la Gestión Asociativa. San Salvador: IICA. 98 p.

ARTARAZ, M. 2001. Teoría de las tres dimensiones de desarrollo sostenible. Ecosistemas. 10(3):1 - 6 .

ASOHOFRUCOL. ASOCIACIÓN HORTIFRUTÍCOLA DE COLOMBIA. 2012. Plan Nacional de Fomento Hortifrutícola 2012 - 2022. Disponible en: http://www.asohofrucol. com.co/archivos/biblioteca/biblioteca_204_Dr. Alvaro Ernesto Palacio Pelaez.pdf; consulta: marzo, 2015.

BIJMAN, J. 2013. Las cooperativas agroalimentarias en la UE. Empresas modernas con retos modernos. Mediterráneo Económico. Colección Estudios Sociales. $24: 41-60$.

BOLAÑOS, 0. 1999. Caracterización y tipificación de organizaciones de productores y productoras. 31 - 39. En: XI Congreso Nacional Agronómico/I Congreso Nacional de Extensión. Costa Rica.

CENTRO PARA EL DESARROLLO DE CAPITAL HUMAN0. 2007. Desarrollo de una metodología de fomento de la asociatividad del sector micro y pequeño empresarial. Santiago: CENDEC. 145 p.

CONGRESO DE COLOMBIA. 1994. Ley 118 de 1994. Disponible en: http://www.asohofrucol.com.co/archivos/ fnfh_marcolegal_1_anexo13.pdf; consulta: marzo, 2015.

EFENDIEV, A.; SOROKIN, P. 2013. Rural Social Organization and Farmer Cooperatives Development in Russia and other Emerging Economies: Comparative Analysis. Developing Country Studies. 3(14):106 - 116.

GOTTRET, M. V.; JUNKIN, R.; UGARTE, C. I. 2011. Autoevaluación facilitada para la gestión de empresas Asociativas rurales. Turrialba, Costa Rica: Centro Agronómico Tropical de Investigación y Enseñanza. 48 p.

IICA. INSTITUTO INTERAMERICANO DE COOPERACIÓN PARA LA AGRICULTURA. 2009. Estudios de caso en comercialización de pequeños productores rurales. En: 
http://legacy.iica.int/Esp/organizacion/LTGC/ agroindustria/Documentos\%20Agroindustria $\% 20$ Rural/Estudios $\% 20$ de $\% 20$ cas o $\% 20$ en $\% 20$ c o mercializaci \% C $3 \%$ B 3 n \% 20 d e \% 20 peque $\%$ C $3 \%$ B1 os $\% 20$ productores $\% 20$ rurales. pdf;consulta: mayo, 2015.

LAMNEK, S. 2005. Qualitative social research. Cuarta edición. Holz. Berlin. 191 p.

LATTUADA, M.; NOGUEIRA, M. E.; RENOLD, J. M.; URCOLA, M. 2011. El cooperativismo agropecuario argentino en la actualidad. Presentación y análisis de tres casos desde la perspectiva del capital social. Mundo Agrario. 12(23):1 - 37.

MARTÍNEZ, P. 2006. El método de estudio de caso: Estrategia metodológica de la investigación científica. Pensamiento y Gestión. 20:165 - 193.

MEDICI, A. 2006. Empreendedorismo e associativismo nos agronegócios: a sua presença (e ausência) na região de Botucatu-Sp. Revista Ciência Em Extensão. 3(1):70 - 80 .

MELGAREJO, Z.; VERA, M. A.; MORA, E. H. 2013. Competitividad de la MIPYME y desarrollo regional. Estudio del caso colombiano. Tendencias. 14(2):184 -215.

MORENO, D.; URIBE, M. C.; SANTIAGO, L. F. 2011. Comunicación y manejo social para la asociatividad: Mecanismo para la sostenibilidad rural. Bogotá: SENA-SAC. 28 p.

NOELLE-NEUMANN, E.; PETERSEN, T. 2005. Alle, nicht jeder. Einführung in die Methoden der Demoskopie. Cuarta edición. Springer-Verlag. Berlin. 660 p.

OSTERTAG, C. F. 2002. Factores claves de éxito de una agroindustria rural : el caso de la asociación de producción y mercadeo para la educación - ASPROME, Cali, Colombia. FA0, Oficina Regional para América Latina y el Caribe. Santiago de Chile, 27 p.

PLATA, J.; GUTIERREZ, Y.; GUTIERREZ, Y. L. 2010. El impacto de los parafiscales en el sector agropecuario. Revista Desarrollo Gerencial. (2):118 - 142.

RIELLA, A.; VITELLI, R. 2009. Organizaciones rurales y acción colectiva en Uruguay : estudios en tiempos de crisis. Primera edición. Europe Aid Co-operation Office. Montevideo. 306 p.

RINCÓN, N.; SEGOVIA, E.; AGUILERA, G.; LÓPEZ, A.; ZAVARCE, E.; LEAL, M. 2004. Los pequeños productores y su participación en el proceso de comercialización agrícola. Revista de La Facultad de Agronomía. 21(2):1 - 13.

RODRIGUEZ, H.; GARCÍA, R.; IWAMOTO, I. 2008. The reasons for failure in the regional plan of agricultural marketing in Colombia: The case of the fruit and vegetables marketing plan in the Cauca Valley Province. Agricultural Marketing Journal. 17(1):32 - 40.

RUEDA, M. A.; MUÑOZ, J. H. 2011. Asociatividad, capital social y redes de innovación en la economía rural. Gestión Social. 4(1):27 - 41.

SEPÚLVEDA, S.; CHAVARRÍA, H.; CASTRO, A.; ROJAS, P.; PICADO, E.; BOLAÑOS, D. 2002. Metodología para estimar el nivel de desarrollo sostenible en espacios territoriales. En: http://ecaths1.s3.amazonaws.com/ desarrollosostenible/176848813.Metodologia\%20estimar\%20DS\%20territorio.PDF; consulta: noviembre, 2015.

SOCIEDAD DE AGRICULTORES DE COLOMBIA. 2010. Sector Rural y Agropecuario: Desarrollo y Futuro. En: http://www.fedearroz.com.co/noticias-docs/POLITICA_SECTORIAL_SAC.pdf; consulta: mayo, 2015.

SZMULEWICZ, P.; GUTIÉRREZ, C.; WINKLER, K. 2012. Evaluación de las habilidades asociativas en redes de agroturismo del sur de Chile. Estudios y Perspectivas en Turismo. 21:1013 - 1034. 